\title{
Suspension technique for reconstructing temporal lower eyelid defects
}

\author{
Wendell B Whitacre MD, Albert Chi MD
}

A vulsion of skin from the temporal aspect of the lower eyelid secondary to bicycle or motorcycle accidents is frequent enough to suggest the appellation of 'cyclist eyelid'. Defects in the same area from trauma, in general, and from excision of benign and malignant lesions are not uncommon.

The time-honoured method for dealing with such wounds has been a full-thickness graft or, at times, a local flap. Over the past several years, the senior author has used a suspension technique.

Following any necessary debridement, the defect is converted into an ellipse or crescent - the long axis of which parallels natural skin wrinkles. Some undermining of the inferior margin of the wound may be appropriate. A small incision is then made in the superior temporal margin of the upper eyelid and carried down to the supraorbital rim. The temporal fascia is exposed and a tract is developed bluntly at the periosteal

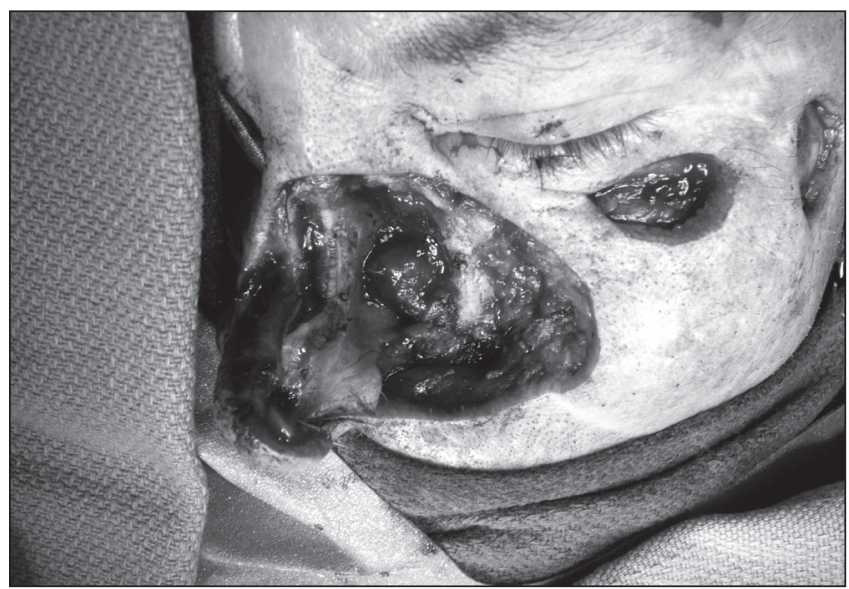

Figure 1) A 50-year-old man following excision of basal cell carcinomas of the nose, left lower eyelid and left zygomatic area level between the two eyelid wounds. The lower eyelid wound is then closed by suspending its central-inferior margin to the temporal fascia with two 4-0 clear nylon sutures. The sutures are passed through the previously developed tunnel. Both sutures should be placed before either is tied. The upper eyelid wound is closed with a 5-0 clear nylon suture in the deeper tissues, and the skin in both areas is closed with a 6-0 black nylon suture. The patient should be warned that there will be an indentation in the lower eyelid scar for a few weeks.

Obviously, this type of repair is similar to suspension of the central soft facial tissues following craniofacial exposure. The technique provides good to satisfactory outcomes, requires less time than a graft and results in better cosmesis.

Perhaps other surgeons have used the same suspension technique. However, no reports of this approach could be found in the literature.

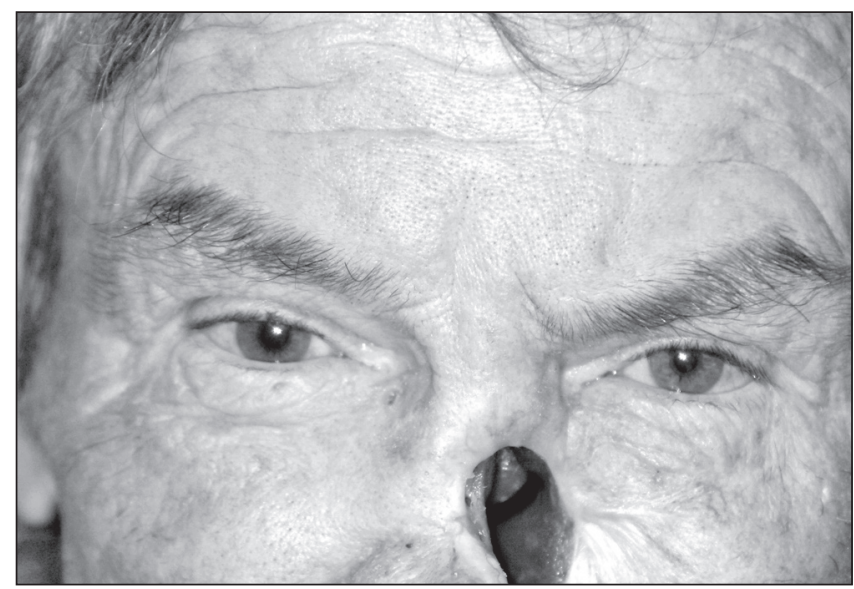

Figure 2) Result 22 months later following repair of lower eyelid defect using the suspension technique. Nose since reconstructed by Dr Frederick Menick 